\title{
METODOLOGIA ATIVA NO ESTÁGIO SUPERVISIONADO DE ENFERMAGEM: INOVAÇÃO NA ATENÇÃO PRIMÁRIA À SAÚDE
}

\author{
ACTIVE METHODOLOGY IN NURSING \\ SUPERVISED INTERNSHIP: INNOVATION IN \\ PRIMARY HEALTH CARE
}

\section{METODOLOGÍA ACTIVA EN LA PASANTÍA SUPERVISADA EN ENFERMERÍA: INNOVACIÓN EN LA ATENCIÓN PRIMARIA DE SALUD}

\author{
Gabriela de Alencar Veiga ${ }^{1}$ \\ Mayssa da Conceição Araújo ${ }^{2}$ \\ Fernanda Letícia Frates Cauduro ${ }^{3}$ \\ Juliane Andrade ${ }^{4}$
}

\begin{abstract}
Como citar este artigo: Veiga GA, Araújo MC, Cauduro FLF, Andrade J. Metodologia ativa no estágio supervisionado de enfermagem: inovação na Atenção Primária à Saúde. Rev baiana enferm. 2020. 34:e34857.

Objetivo: relatar a experiência do uso de uma metodologia ativa no estágio supervisionado na Atenção Primária à Saúde. Método: trata-se de um relato de experiência sobre o uso de uma metodologia ativa no estágio supervisionado, realizado entre março e junho de 2019, baseado nos pressupostos da educação problematizadora de Paulo Freire. Como estratégia pedagógica utilizou-se a Metodologia da Problematização, apoiada no Arco de Maguerez. Resultados: o Arco de Maguerez possibilitou implantar ações de educação em saúde na sala de vacina da unidade de saúde. Este processo de ensino-aprendizagem viabilizou uma postura ativa das acadêmicas envolvidas, convergiu para o propósito de facilitação da professora e resultou na efetiva implementação das estratégias planejadas durante a execução do Arco. Conclusão: o presente relato expôs uma experiência exitosa no âmbito do ensino-aprendizagem, de forma a protagonizar o papel das acadêmicas, em consonância com as diretrizes curriculares que indicam prática pedagógica ética, reflexiva e transformadora.
\end{abstract}

Descritores: Atenção Primária à Saúde. Enfermagem. Aprendizagem Baseada em Problemas.

Objective: to report the experience of using an active methodology in the supervised internship in Primary Health Care. Method: this is an experience report on the use of an active methodology in the supervised internship, conducted between March and June 2019, based on assumptions of problem-based education of Paulo Freire. The pedagogical strategy used was the Problem-Based Methodology, supported by the Arch of Maguerez. Results: the Arch of Maguerez enabled deploying health education actions in the vaccination room of the health unit. This teaching-learning process allowed for an active behavior of the students involved, converged to the professor's facilitation and resulted

\footnotetext{
Enfermeira. Residente em Gestão de Políticas Públicas em Saúde da FioCruz. Brasília, Distrito Federal, Brasil. https://orcid.org/0000-0002-7348-7249.

Enfermeira. Residente em Enfermagem na Saúde do Adulto e do Idoso da Escola de Enfermagem da Universidade de São Paulo. São Paulo, SP, Brasil. https://orcid. org/0000-0002-8475-6950.

Enfermeira. Doutora em Ciências. Professora Adjunta da Universidade de Brasília. Brasília, Distrito Federal, Brasil. https://orcid.org/0000-0002-473|-9217.

Enfermeira. Doutora em Saúde Coletiva. Professora Adjunta da Universidade de Brasília. Brasília, Distrito Federal, Brasil. juenf andrade@yahoo.com.br. https://orcid. org/0000-0002-4321-0118.
} 
in the effective implementation of planned strategies during the implementation of the Arch. Conclusion: the present report outlined a successful experience in the context of the teaching-learning process, in order to provide the students the main role, in line with the curricular guidelines that indicate ethical, reflective and transformative pedagogical practices.

Descriptors: Primary Health Care. Nursing. Problem-Based Learning.

Objetivo: reportar la experiencia de utilización de una metodología activa en la pasantía supervisada en la Atención Primaria de Salud. Método: se trata de un relato de experiencia en el uso de una metodología activa en la pasantía supervisada, realizado entre marzo y junio de 2019, con base en supuestos de educación basada en problemas de Paulo Freire. Como estrategia pedagógica, se utilizó la Metodología Basada en Problemas, con base en el Arco de Maguerez. Resultados: el Arco de Maguerez permitió implementar acciones en educación para la salud en la sala de vacunación de la unidad de salud. Este proceso de enseñanza-aprendizaje posibilitó una postura activa de las estudiantes involucradas, convergió con el propósito de facilitación del profesor y se tradujo en la aplicación eficaz de estrategias planeadas durante la ejecución del Arco. Conclusión: el presente informe reporta una experiencia exitosa en el contexto del proceso de enseñanza-aprendizaje, con el fin de protagonizar el papel de las estudiantes, en línea con las directrices curriculares que indican las prácticas pedagógicas éticas, reflexivas y transformadoras.

Descriptores: Atención Primaria de Salud. Enfermería. Aprendizaje Basado en Problemas.

\section{Introdução}

O processo de ensino-aprendizagem é dinâmico e está em constante transformação. Ensinar na era das comunicações ultra rápidas e das fake news demanda métodos inovadores que admitam uma prática pedagógica ética, crítica, reflexiva e transformadora ${ }^{(1)}$. É neste cenário que as metodologias ativas ganham espaço e reconhecimento.

A metodologia de ensino-aprendizagem comumente aplicada nos cursos da área da saúde remete ao modelo tradicional ${ }^{(1)}$, com a transferência de conhecimento docente-discente, supervalorização da técnica e distanciamento do contexto social $^{(2)}$. Sair da formação tradicional é um desafio no ensino dos cursos de saúde. A falta de infraestrutura dos serviços de saúde e das instituições de ensino pode colocar em risco a prática educativa, tornando-a desestimulante ${ }^{(3)}$.

As diretrizes curriculares dos cursos da área da saúde contemplam mudanças paradigmáticas, ao determinarem que as universidades devem estimular a articulação entre ensino, pesquisa e assistência, o que remete à inovação e à qualidade do projeto político-pedagógico ${ }^{(1)}$. $\mathrm{Na}$ enfermagem, os estudantes necessitam refletir sobre a prática do cuidado, comprometer-se com as necessidades da população e, por meio da problematização da realidade, buscar compreender, explicar e transformar sua práxis ${ }^{(4)}$.

Nesse cenário, surgem as metodologias ativas (MA) para apoiar o processo de ensino-aprendizagem, ao propiciarem momentos que ampliam a reflexão e a criticidade sobre o objeto estudado $^{(1)}$. Nesse contexto, o educando é sujeito ativo no seu aprendizado. Assim, tem-se, nas MA, um caminho para promover a relação ensino-pesquisa-assistência e potencializar a formação de profissionais com o olhar atento às demandas da sociedade.

As MA têm sido utilizadas em algumas disciplinas do curso de Enfermagem da Universidade de Brasília (UnB), inclusive nos estágios supervisionados na Atenção Primária à Saúde (APS). Elas têm favorecido a aproximação do acadêmico com demandas reais da equipe de saúde e dos usuários.

O estágio supervisionado contempla uma etapa obrigatória na formação do acadêmico e futuro enfermeiro e pode ser executado em hospitais, serviços especializados, ambulatórios e na APS $^{(5)}$. Nesses cenários, o aluno amadurece seu papel profissional, ao vincular a teoria e a prática. Além disso, são promotores de inovação no local, pois o aluno vale-se de conhecimentos 
atualizados que reverberam positivamente nas ações e intervenções por ele executadas ${ }^{()}$.

Face ao exposto, o objetivo do presente artigo é relatar a experiência do uso de uma MA no estágio supervisionado na APS.

\section{Método}

Trata-se de um relato de experiência sobre o uso de uma MA no estágio supervisionado. Participaram da atividade cinco acadêmicas do penúltimo semestre do curso de Enfermagem da UnB, inscritas na disciplina Estágio Supervisionado na APS. Dentre os objetivos dessa disciplina destaca-se produzir e implementar uma intervenção no cenário, com base em necessidades identificadas no serviço.

As ações foram realizadas entre maio e junho de 2019 em uma Unidade Básica de Saúde (UBS) de Brasília (DF), com cobertura de 42 mil usuários. À época, a UBS era formada por quatro equipes multiprofissionais de Saúde da Família e odontologia. As equipes, entretanto, não contavam com agentes comunitários de saúde (ACS), o que restringe o cálculo real da área de abrangência. O modelo de atenção à saúde no DF encontra-se em transição, conforme Portaria n. 77 , de $2017^{(7)}$, da Secretaria do Estado de Saúde do Distrito Federal.

Para as ações de educação em saúde, o referencial teórico foi constituído pelos pressupostos da educação problematizadora de Paulo Freire, segundo a qual o saber, o ensinar e o aprender devem ser vistos como instrumentos horizontais e multilaterais de transmissão de saberes, e o conhecimento deve ser construído por condições e possibilidades de diálogo entre os indivíduos ${ }^{(2,8)}$. A estratégia pedagógica adotada foi a Metodologia da Problematização, apoiado no Arco de Maguerez ${ }^{(9)}$.

O Arco de Maguerez é constituído por cinco etapas, a saber:

a) observação da realidade: consiste na observação de uma determinada realidade, registro das fragilidades presenciadas e eleição daquela que impacta no processo de trabalho local. O tempo para execução, a governabilidade e a articulação dos atores envolvidos na realidade são variáveis a serem consideradas nesta etapa;

b) pontos-chave: prevê a seleção de elementos que facilitam a compreensão do problema elencado;

c) teorização: com base na etapa anterior, são consultados referenciais científicos, a fim de consolidar o conhecimento e fornecer subsídios para a formulação de hipótese para solucionar o problema;

d) hipóteses de solução: elencam-se propostas aplicáveis à realidade e são selecionadas aquelas que produzirão impacto significativo no contexto analisado;

e) aplicação à realidade: são implementadas soluções factíveis à realidade com base no processo ação-reflexão-ação.

\section{Resultados}

Na disciplina de Estágio Supervisionado, os acadêmicos são mobilizados a vivenciar a realidade da UBS, problematizar as situações assistenciais e gerenciais do campo de estágio e propor intervenções que agreguem valor aos profissionais e usuários do serviço de saúde. Nesse sentido, a MA escolhida buscou tornar o ambiente de ensino construtivo, colocando o acadêmico no centro do processo na elaboração de ações inovadoras, respaldadas nas etapas do Arco de Maguerez, como descrito a seguir:

\section{Primeira etapa - Observação da realidade}

A observação da realidade ocorreu no mês de março de 2019, e as acadêmicas foram orientadas a observar o processo de trabalho da UBS. Diversas fragilidades foram listadas, sendo as principais: ausência de ACS, com consequente ausência de territorialização; ações da unidade extra muro, como a visita domiciliar; desorganização do carrinho de emergência (falta de medicações, checagem e lacre); falta de capacitação/manejo no acolhimento dos usuários; dificuldade no gerenciamento de recursos materiais; e carência de ações de educação em saúde quanto à imunização (recomendações, faixa 
etária, doenças prevenidas) e à ambiência na sala de vacina, considerando o público infantil.

A seleção de uma fragilidade considerou a governabilidade das acadêmicas e o tempo para a execução da intervenção. Assim, elegeu-se realizar ações na sala de vacina, com a participação ativa dos profissionais de saúde e do gestor da UBS. Tal escolha também foi influenciada pelo contexto nacional da baixa cobertura vacinal. Um dos fatores é o fortalecimento dos movimentos anti-vacinas, refletidos nos casos de sarampo registrados, o que salienta a importância da comunicação e do vínculo da população com as ações de vacinação ${ }^{(10)}$.

Dessa maneira, após a caracterização da sala de vacina, as acadêmicas, apoiadas em referencial teórico, exploraram possibilidades de intervenção, a fim de minimizar as fragilidades constatadas. Nesse aspecto, foram prospectadas possíveis ações de educação em saúde.

Isto posto, foi adotado o conceito de educação em saúde do Ministério da Saúde, o qual afirma ser essa o "[...] conjunto de práticas do setor que contribui para aumentar a autonomia das pessoas no seu cuidado e no debate com os profissionais e os gestores a fim de alcançar uma atenção à saúde de acordo com suas necessidades"(11:19-20).

\section{Pontos-chave e teorização}

Com vistas a articular os resultados da segunda e terceira etapas do Arco de Maguerez, o Quadro 1 apresenta a síntese dos registros produzidos pelas acadêmicas de enfermagem.

Quadro 1 - Pontos-chave e teorização com base na identificação da carência de ações de educação em saúde quanto à imunização e ambiência na sala de vacina

(continua)

\begin{tabular}{|c|c|c|}
\hline \multirow{2}{*}{ Pontos-chave } & \multicolumn{2}{|r|}{ Teorização } \\
\hline & Referência & Conteúdo \\
\hline $\begin{array}{l}\text { A educação em saúde, } \\
\text { atribuição de qual } \\
\text { profissional? }\end{array}$ & $\begin{array}{l}\text { Barreto ACO, Rebouças } \\
\text { CBA, Aguiar MIF, } \\
\text { Barbosa RB, Rocha SR, } \\
\text { Cordeiro LM, et al }{ }^{(12)}\end{array}$ & $\begin{array}{l}\text { Os autores trazem que a Educação em Saúde é } \\
\text { uma atribuição da equipe multiprofissional da } \\
\text { APS, mas destaca o enfermeiro como importante } \\
\text { educador, mesmo de forma indireta, pela } \\
\text { atribuição do gerenciamento da UBS. }\end{array}$ \\
\hline \multirow[t]{3}{*}{$\begin{array}{l}\text { Como facilitar aos } \\
\text { usuários o acesso à } \\
\text { informação na APS? }\end{array}$} & Freire $\mathrm{P}^{(2)}$ & $\begin{array}{l}\text { A produção de conhecimento é oportunizada } \\
\text { pela relação do educando com o meio no qual } \\
\text { está inserido. São consideradas as questões } \\
\text { histórico-sociais e as novas formas de conhecer } \\
\text { e interpretar o mundo são propiciadas pelo } \\
\text { apoio de um facilitador. }\end{array}$ \\
\hline & Brasil $^{(13)}$ & $\begin{array}{l}\text { Prevê a realização de ações de educação em } \\
\text { saúde destinadas à população do território } \\
\text { de abrangência da equipe, com o uso de } \\
\text { metodologias participativas e emancipatórias. }\end{array}$ \\
\hline & $\begin{array}{l}\text { Duarte DC, Oliveira VC, } \\
\text { Guimarães EAA, } \\
\text { Viegas SMF }^{(14)}\end{array}$ & $\begin{array}{l}\text { Apontam sentidos e sentimentos de usuários frente } \\
\text { à vacinação; indicam a necessidade de reforçar a } \\
\text { comunicação efetiva entre esses e os serviços de } \\
\text { saúde para ampliação da cobertura vacinal. }\end{array}$ \\
\hline Para que imunizar? & Brasil $^{(15)}$ & $\begin{array}{l}\text { As vacinas estimulam o sistema imunológico a } \\
\text { desenvolver proteção à pessoa contra doenças } \\
\text { transmissíveis, de forma segura. É uma importante } \\
\text { estratégia de saúde pública, visto a magnitude de } \\
\text { seus efeitos no processo saúde-doença. }\end{array}$ \\
\hline
\end{tabular}


Quadro 1 - Pontos-chave e teorização com base na identificação da carência de ações de educação em saúde quanto à imunização e ambiência na sala de vacina

(conclusão)

\begin{tabular}{|l|l|l|}
\hline \multirow{2}{*}{ Pontos-chave } & \multicolumn{2}{|c|}{ Teorização } \\
\cline { 2 - 3 } & \multicolumn{1}{|c|}{ Referência } & \multicolumn{1}{c|}{ Conteúdo } \\
\hline $\begin{array}{l}\text { Como se apresenta o } \\
\text { calendário vacinal do } \\
\begin{array}{l}\text { DF referente ao ano } \\
\text { de 2019? }\end{array}\end{array}$ & Distrito Federal & $\begin{array}{l}\text { Apresenta o calendário por faixa etária, com } \\
\text { observações quanto às particularidades de cada } \\
\text { vacina, se necessário. }\end{array}$ \\
\hline $\begin{array}{l}\text { Como promover a } \\
\text { ambiência na UBS? }\end{array}$ & Brasil $^{(15)}$ & $\begin{array}{l}\text { Propicia espaços físicos humanizados e } \\
\text { acolhedores, segundo as necessidades dos } \\
\text { usuários. }\end{array}$ \\
\hline
\end{tabular}

Fonte: Elaboração própria.

Hipóteses de Solução e Aplicação à

Realidade

Como desfecho das etapas anteriores, as acadêmicas, professora e equipe de saúde propuseram a construção da "Árvore da Vacinação" como solução para a carência de informações sobre vacinas; e o "Jardim da Vacinação" - como solução para a carência de estratégias para ambiência da sala de vacinação.

O protótipo consistiu em uma árvore cujos frutos são quadros que apresentam as vacinas a serem recomendadas em cada faixa etária. Foram elaborados 12 quadros, com elementos lúdicos e as informações: idade indicada, nome do imunobiológico, indicação de proteção, imagem ilustrativa da faixa etária e fonte oficial (Figura 1).

Figura 1 - Modelo do quadro informativo sobre as vacinas recomendadas ao nascer e as doenças prevenidas

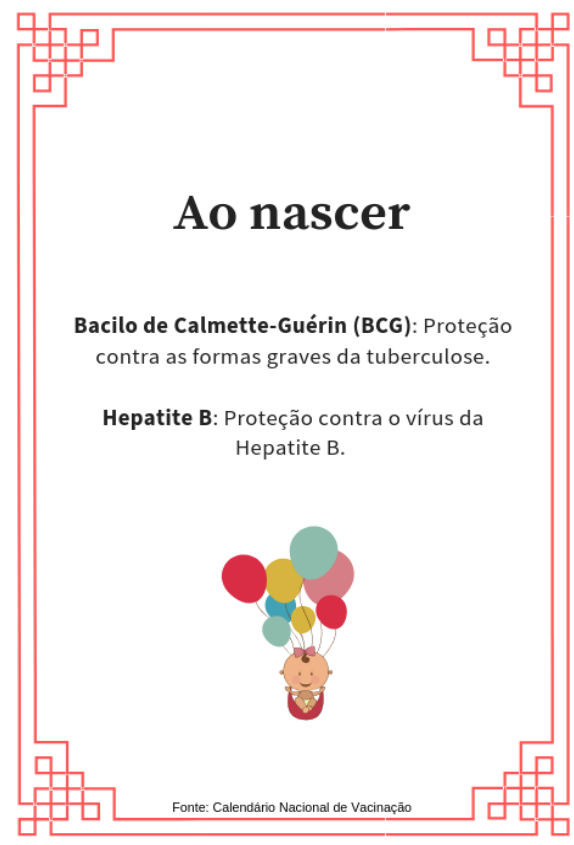

Fonte: Elaboração própria. 
Para apresentação dos quadros de forma atrativa, sequencial e informativa, duas árvores foram construídas; a primeira, refletia o calendário vacinal infantil; a segunda, representava as exceções, como gestantes, adultos e idosos. Para a ambiência, utilizaram-se tampas dos frascos de imunobiológicos para elaborar a identificação da sala de vacina. No "Jardim da Vacinação", aplicaram-se imagens auto-adesivas, adquiridas pelas acadêmicas e professora, com o objetivo de ressignificar o ambiente (Figura 2).

Figura 2 - Árvore da Vacinação e Jardim da Imunização

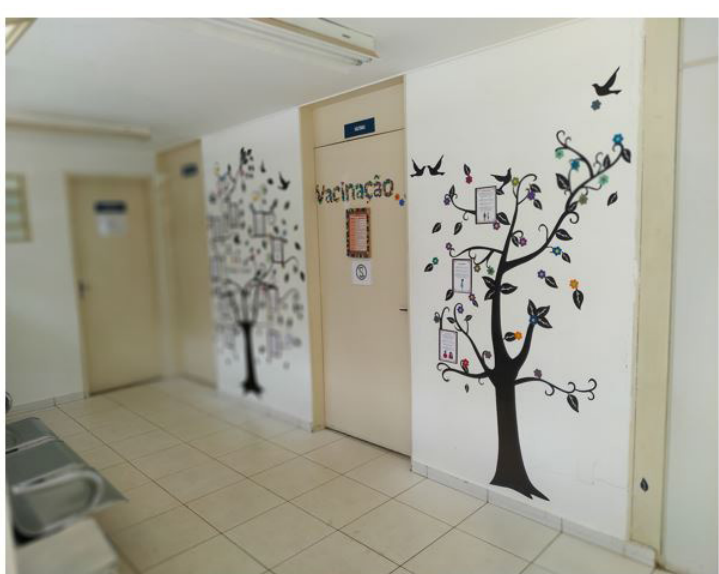

Fonte: Arquivos pessoais.

Após a implementação, os usuários elogiaram a iniciativa e comentaram sobre a árvore com os profissionais de saúde, além de serem vistos observando a árvore e conferindo o próprio cartão vacinal. O processo de construção e aplicação à realidade das estratégias planejadas possibilitou uma interação significativa entre profissionais, gestores, usuários do serviço e acadêmicas.

Ao final do curso de graduação, o acadêmico de enfermagem passou por momentos de aprendizagem teórica e prática, percorreu as etapas de observação, descrição de cenários, demonstração e, ao adentrar no estágio supervisionado, respondeu aos estímulos do cenário de prática com a execução de tarefas que poderão compor, de forma habitual, sua práxis.

O estágio supervisionado prevê a integração entre academia e campo profissional, aproxima o acadêmico de situações reais e oportuniza a efetiva mobilização de conhecimentos, habilidades e atitudes aprendidas durante o ciclo de formação. Seu objetivo é possibilitar ao acadêmico articular teoria e prática, por meio de

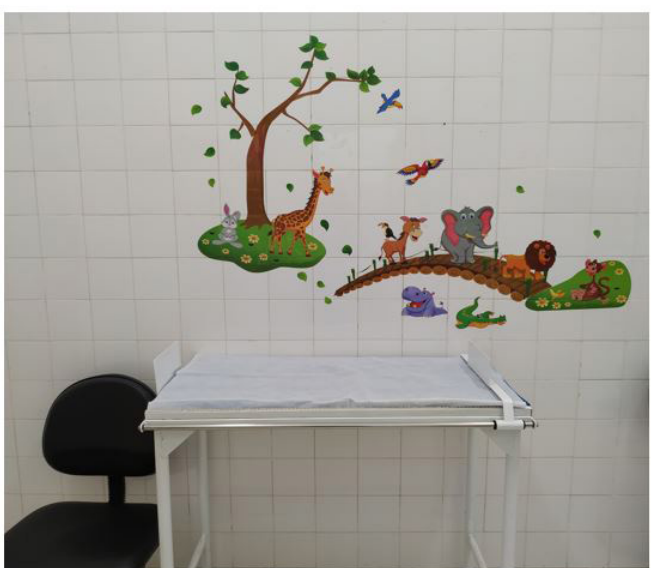

estratégias que ampliem habilidades de comunicação, flexibilidade e tomada de decisão ${ }^{(17-18)}$.

O uso de MA no estágio supervisionado potencializa a autonomia do acadêmico e favorece a lapidação de competências. Na experiência relatada, o Arco de Maguerez e suas etapas foram realizados em totalidade e possibilitaram a criação de intervenções criativas e inovadoras, considerando o contexto, profissionais e usuários do serviço de saúde.

A literatura aponta que, entre as MA aplicadas em estágio supervisionado, a Metodologia da Problematização e o Arco são referência, pois almeja-se a resolução de problemas factíveis e há ressignificação de conhecimentos respaldados pela pedagogia problematizadora de Paulo Freire ${ }^{(18)}$.

Com relação ao Arco, sua primeira versão foi descrita pelo próprio Maguerez, porém é com base nos escritos de Bordenave e Pereira que se vislumbra a disseminação do seu potencial de uso no ensino. Nos ensaios produzidos pelos autores, a observação do concreto, seguida pelo estudo, reflexão teórica e retorno à realidade 
com possibilidades de intervenção, levando-se em consideração o envolvimento de todos os atores (produtores e consumidores da intervenção), apontam para ações que geram ressignificação e transformação de realidades ${ }^{(19)}$.

Tais potencialidades têm sido objeto de investigação no ensino em saúde, seja na APS, seja em contextos de maior complexidade. Nesse aspecto, em estudo no qual a Metodologia da Problematização foi utilizada em disciplina teórico-prática na APS, as autoras identificaram que a estratégia atendeu aos objetivos pedagógicos e possibilitou a construção de competências necessárias para o trabalho do enfermeiro no Sistema Único de Saúde ${ }^{(20)}$.

Destarte, a referida metodologia, apoiada no Arco de Maguerez, também tem auxiliado acadêmicos de enfermagem durante o estágio supervisionado na APS, no desenvolvimento do raciocínio clínico e na execução do processo de enfermagem, o que demonstra seu caráter multifacetado e aplicabilidade em situações assistenciais, gerenciais e organizacionais ${ }^{(19)}$.

Nesse contexto, também é importante refletir sobre o papel do docente que, ao utilizar estratégias didático-pedagógicas emancipatórias, precisa constantemente qualificar sua prática pedagógica e apoiá-la em referenciais teóricos e epistemológicos. O direcionamento teórico favorece a ação do docente que, por vezes, reproduz, de forma empírica, estratégias vivenciadas em sua formação ${ }^{(21)}$. Assim, a MA a ser aplicada deve ir ao encontro de objetivos de aprendizagem pré-definidos, convergentes para o objetivo do estágio supervisionado, a fim de possibilitar ao acadêmico a atribuição de significado às atividades por ele desempenhadas.

Nessa seara, há também que se considerar a importância da postura ativa do acadêmico, tendo em vista que a MA visa o seu protagonismo. Na experiência relatada, o engajamento das estudantes convergiu para o propósito de facilitação da professora e resultou na efetiva implementação das estratégias definidas durante a execução do Arco. Essas, além de responderem a uma necessidade do campo de prática, foram ao encontro das atuais demandas do Ministério da Saúde, sobretudo no que concerne à vacinação.
Apregoa-se a necessidade de ações que intensifiquem e ampliem o acesso à informação da população quanto às vacinas, atualização do calendário vacinal e relevância da imunização como dispositivo na prevenção de doenças ${ }^{(22)}$.

Considera-se como limitação da ação realizada a impossibilidade de avaliar a reação dos gestores e dos usuários da UBS frente às novas informações disponibilizadas e as adequações realizadas na sala de vacina, devido ao recorte temporal do estágio supervisionado.

\section{Conclusão}

O presente artigo permitiu relatar uma experiência exitosa no âmbito do ensino-aprendizagem em estágio supervisionado, apoiado por MA, de forma a mostar o protagonismo das acadêmicas de enfermagem. Tal vivência foi relevante, visto ir ao encontro das competências almejadas para o futuro profissional de saúde e estar em consonância com as diretrizes curriculares nacionais dos cursos de graduação em saúde.

\section{Colaborações:}

1 - concepção, projeto, análise e interpretação dos dados: Gabriela de Alencar Veiga, Mayssa da Conceição Araújo, Fernanda Leticia Frates Cauduro e Juliane Andrade;

2 - redação do artigo e revisão crítica relevante do conteúdo intelectual: Gabriela de Alencar Veiga, Mayssa da Conceição Araújo, Fernanda Leticia Frates Cauduro e Juliane Andrade;

3 - aprovação final da versão a ser publicada: Fernanda Leticia Frates Cauduro e Juliane Andrade.

\section{Referências}

1. Colares KTP, Oliveira W. Metodologias Ativas na formação profissional em saúde: uma revisão. Rev SUSTINERE. 2018 jul-dez;6(2):300-20. DOI: http:// dx.doi.org/10,12957/sustinere.2018.36910

2. Freire P. Pedagogia da Autonomia: saberes necessários à prática educativa. São Paulo: Paz e Terra; 1996. 
3. Sobral FR, Campos CJG. Utilização de metodologia ativa no ensino e assistência de enfermagem na produção nacional: revisão integrativa. Rev Esc Enferm USP [online]. 2012;46(1):208-18. DOI: https://doi.org/10.1590/S0080-62342012000100028

4. Villardi ML, Cyrino EG, Berbel NAN. A metodologia da problematização no ensino em saúde: suas etapas e possibilidades. In: Villardi ML, Cyrino EG, Berbel NAN. A problematização em educação em saúde: percepções dos professores tutores e alunos [Internet]. São Paulo: EdUNESP; Cultura Acadêmica; 2015. p. 45-52. [cited 2019 Dec 10]. Available from: http://books.scielo.org/id/dgjm7/ pdf/villardi-9788579836626-05.pdf

5. Brasil. Ministério da Educação. Conselho Nacional de Educação. Câmara de Educação Superior. Resolução CNE/CES n. 3, de 7 de novembro de 2001. Institui Diretrizes Curriculares Nacionais do Curso de Graduação em Enfermagem [Internet]. Brasília (DF); 2001 [cited 2019 Dec 10]. Available from: http://portal.mec.gov.br/cne/arquivos/pdf/ CES03.pdf

6. Benito GAV, Tristão KM, Paula ACSF, Santos MA, Ataide LJ, Lima RCD. Desenvolvimento de competências gerais durante o estágio supervisionado. Rev Bras Enferm. 2012 Jan-Feb;65(1):172-8. DOI: https://doi.org/10.1590/S0034-71672012000100025

7. Distrito Federal. Secretaria do Estado de Saúde do Distrito Federal. Portaria n. 77, de 14 de fevereiro de 2017. Estabelece a Política de Atenção Primária à Saúde do Distrito Federal [Internet]. Brasília (DF); 2017 [cited 2019 Dec 10]. Available from: http://www.saude.df.gov.br/wp-conteudo/ uploads/2018/04/Portaria-SES_DF-n\%C2\%BA-772017-Esstabelece-a-Pol\%C3\%ADtica-de-Aten $\%$ C3\%A7\%C3\%A3o-Prim\%C3\%A 1ria-\%C3\%A 0Sa\%C3\%BAde-do-Distrito-Federal.pdf

8. Pitano SC. A educação problematizadora de Paulo Freire, uma pedagogia do sujeito social. Inter-Ação. 2017;42(1):87-104. DOI: http://dx.doi.org/10.5216/ ia.v42i1. 43774

9. Berbel NAN. A utilização da Metodologia da Problematização com o Arco de Maguerez no Cuidar em Saúde. In: França FCV, Melo MC, Monteiro SNC, Guilhem D, organizadores. O processo de ensino aprendizagem de profissionais de saúde: a metodologia da problematização por meio do Arco de Maguerez. Brasília (DF): Universidade de Brasília; 2016. p. 102-19.

10. Sato APS. Qual a importância da hesitação vacinal na queda das coberturas vacinais no Brasil? Rev
Saude Publica [online]. 2018;52:96. DOI: https:// doi.org/10.11606/s1518-8787.2018052001199

11. Brasil. Ministério da Saúde. Secretaria-Executiva. Secretaria de Gestão do Trabalho e da Educação na Saúde. Glossário temático: gestão do trabalho e da educação na saúde [Internet]. 2a ed. Brasília (DF); 2012 [cited 2019 Dec 10]. (Série A. Normas e Manuais Técnicos). Available from: http://bvsms.saude.gov.br/bvs/ publicacoes/glossario_tematico_gestao_trabalho_ educacao_saude_2ed.pdf

12. Barreto ACO, Rebouças CBA, Aguiar MIF, Barbosa RB, Rocha SR, Cordeiro LM, et al. Percepção da equipe multiprofissional da Atenção Primária sobre educação em saúde. Rev Bras Enferm. 2019;72(Suppl 1):278-85. DOI: https://doi. org/10.1590/0034-7167-2017-0702

13. Brasil. Ministério da Saúde. Portaria no 2.436, de 21 de setembro de 2017. Aprova a Política Nacional de Atenção Básica, estabelecendo a revisão de diretrizes para a organização da Atenção Básica, no âmbito do Sistema Único de Saúde (SUS) [Internet]. Brasília; 2017 [cited 2019 Dec 11]. Available from: http://bvsms.saude.gov. br/bvs/saudelegis/gm/2017/prt2436_22_09_2017. html

14. Duarte DC, Oliveira VC, Guimarães EAA, Viegas SMF. Acesso à vacinação na Atenção Primária na voz do usuário: sentidos e sentimentos frente ao atendimento. Esc Anna Nery. 2019;23(1):e20180250. DOI: https://doi. org/10.1590/2177-9465-ean-2018-0250

15. Brasil. Ministério da Saúde. Secretaria de Vigilância em Saúde. Departamento de Vigilância Epidemiológica. Programa Nacional de Imunizações (PNI): 40 anos [Internet]. Brasília (DF); 2013 [cited 2019 Dec 11]. Available from: http://bvsms.saude.gov.br/bvs/publicacoes/ programa_nacional_imunizacoes_pni40.pdf

16. Distrito Federal. Instrução Normativa. Calendário Nacional de Vacinação do Distrito Federal [Internet]. Brasília (DF); 2019 [cited 2019 Dec 11]. Available from: http://www.saude.df.gov.br/wp-conteudo/ uploads/2018/03/Instru\%C3\%A7\%C3\%A3oNormativa-2019.pdf

17. Marran AL, Lima PG, Bagnato MHS. As políticas educacionais e o estágio curricular supervisionado no curso de graduação em enfermagem. Trab Educ Saúde. 2015 jan/abr;13(1):89-108. DOI: https://doi. org/10.1590/1981-7746-sip00025 
18. Esteves LSF, Cunha ICKO, Bohomol E, Negri EC. O estágio curricular supervisionado na graduação em enfermagem: revisão integrativa. Rev Bras Enferm. 2018;71(suppl 4):1842-53. DOI: https:// doi.org/10.1590/0034-7167-2017-0340

19. França FCV, Melo MC, Monteiro SNZ, Guilhem D. O processo de ensino aprendizagem de profissionais de saúde: a metodologia da problematização por meio do arco de maguerez. Brasília: Universidade de Brasília; Faculdade de Ciências da Saúde; 2016.

20. Clapis MJ, Marques EA, Corrêa AK, Souza MCBM, Borba KP. Problematization methodology in primary healthcare teaching. Rev Bras Enferm. 2018;71(Suppl 4):1671-7. DOI: https://doi. org/10.1590/0034-7167-2017-0157
21. Araújo VABT, Gebran RA, Barros HF. Formação e práticas de docentes de um curso de graduação em enfermagem. Acta Scientiarum. Education. 2016;38(1):69-79. DOI: 10.4025/actascieduc. v38i1.23180

22. Conselho Nacional de Secretarias Municipais de Saúde. Dez passos para ampliar cobertura vacinal [Internet]. Brasília (DF); 2019 out 8 [cited 2019 Dec 11]. Available from: https://www.conasems.org.br/ dez-passos-para-ampliar-cobertura-vacinal/

Recebido: 13 de dezembro de 2019

Aprovado: 12 de fevereiro de 2020

Publicado: 2 de abril de 2020

A Revista Baiana de Enfermagem utiliza a Licença Creative Commons - Atribuição-NãoComercial 4.0 Internacional. https://creativecommons.org/licenses/by-nc/4.0/

Este artigo é de acesso aberto distribuído sob os termos da Licença Creative Commons (CC BY-NC). Esta licença permite que outros remixem, adaptem e criem a partir do seu trabalho para fins não comerciais. Embora os novos trabalhos tenham de lhe atribuir o devido crédito e não possam ser usados para fins comerciais, os usuários não têm de licenciar esses trabalhos derivados sob os mesmos termos. 